\title{
Numerical Study on Wall Temperature Effects on Shock Wave/Turbulent Boundary-Layer Interaction
}

\author{
Xing-Kun Zhu* and Chang-Ping Yu \\ State Key Laboratory of High-Temperature Gas Dynamics, 100190 Beijing, \\ People's Republic of China \\ Fu-Lin Tong \\ China Aerodynamics Research and Development Center, 621000 Mianyang, \\ People's Republic of China \\ and \\ Xin-Liang Li尽 \\ State Key Laboratory of High-Temperature Gas Dynamics, \\ 100190 Beijing, People's Republic of China \\ DOI: $10.2514 / 1 . J 054939$
}

\begin{abstract}
The effect of wall temperature on the size of the separation bubble in the shock wave/turbulent boundary-layer interaction of a $24 \mathrm{deg}$ compression ramp with Mach 2.9 is numerically investigated. The ratios of wall temperature to recovery temperature $T_{w} / T_{r}$ are $0.6,1.14,1.4$, and 2.0 , respectively. To validate the simulation, the statistical results with $T_{w} / T_{r}=1.14$ are tested and the results show a good agreement with theoretical and experimental results. It is shown that wall temperature has a remarkable effect on the size of the separation bubble and the size increases significantly with the increase of wall temperature. Through theoretical analysis, combined with numerical results, we get a semitheoretical formula $L / \delta \propto\left(T_{w} / T_{r}\right)^{0.85}$, in which $L$ and $\delta$ are the length of the separation bubble and the thickness of upstream boundary layer, respectively. The turbulent kinetic energy budgets are also analyzed based on the numerical data, and results show that turbulence kinetic energy is chiefly produced both in the buffer layer and near the shock wave, and turbulent dissipation is mainly in the center of the separation bubble as well as in the nearwall region. It is also shown that the intrinsic compressibility effect is not significant in all these cases.
\end{abstract}

\section{Nomenclature}

$C_{f}=$ skin friction coefficient

$L=$ size of separation bubble

$M_{\infty}=$ freestream Mach number

$\bar{p}_{w}=$ mean wall pressure

$p_{\infty}=$ static pressure

$T_{r}=$ recovery temperature

$T_{w}=$ wall temperature

$T_{\infty}=$ static pressure

$x_{r}=$ reattachment point

$x_{s}=$ separation point

$\delta=$ upstream boundary-layer thickness

$\mu \quad=$ viscosity coefficient

$\rho \quad=$ density

\section{Introduction}

$\mathbf{S}$ HOCK wave/boundary-layer interaction (SBLI) plays an important role in the aerodynamic design of high-speed vehicles, and the study of SBLI becomes a hot topic again with the deep research of scramjet engines in recent years. SBLI has been studied experimentally, theoretically, and numerically for more than 60 years. But it is still far from being solved and still needs more research work [1-5].

Received 5 December 2015; revision received 31 May 2016; accepted for publication 1 June 2016; published online 9 August 2016. Copyright (@ 2016 by the American Institute of Aeronautics and Astronautics, Inc. All rights reserved. Copies of this paper may be made for personal and internal use, on condition that the copier pay the per-copy fee to the Copyright Clearance Center (CCC). All requests for copying and permission to reprint should be submitted to CCC at www.copyright.com; employ the ISSN 0001-1452 (print) or 1533-385X (online) to initiate your request.

* Master Student, Institute of Mechanics, Chinese Academy of Sciences.

${ }^{\dagger}$ Assistant Professor, Institute of Mechanics, Chinese Academy of Sciences.

¥Assistant Professor.

\$Professor, Institute of Mechanics, Chinese Academy of Sciences; lixl@ imech.ac.cn (Corresponding Author).
Recently, the effect of wall temperature on SBLI has become the focus of attention in the field of hypersonic engineering. During the hypersonic aircraft design process, in general, the temperature of windtunnel tests is usually far different from that of the flight conditions, and engineers want to know more about the effect of wall temperature on SBLI to predict the flight data based on their wind-tunnel tests.

Marini [6] conducted experimental studies about the effects of ramp angle, wall temperature, and Mach number on the scale of separation bubble in shock wave/laminar boundary layer interaction (SLBLI), and then gave some empirical formulas, and these formulas were then assessed numerically by John and Kulkarni [7]. Compared with SLBLI, shock wave/turbulent boundary-layer interaction (STBLI) is more complicated and the study of STBLI is more novel. Jaunet et al. [8] studied experimentally the wall-temperature effects on the STBLI. An adiabatic-wall and two hot-wall cases in reflected STBLI were studied with the ratio of wall temperature to recovery temperature $T_{w} / T_{r}$ are $1,1.4$, and 1.9 , respectively. A low-wall-temperature compression corner STBLI can also be done, with the ratio of temperature to recovery temperature $T_{w} / T_{r}$ being 0.47 . Spaid and Frishett [9] performed an experimental study on the effects of wall temperature on the separation length for a compressible corner with freestream Mach number 2.9. Both experiments [ $[8,9]$ show that the higher the wall temperature, the greater the separation bubble is. Adams [10], Wu and Martín [11,12], and Li et al. [13] used direct numerical simulation (DNS) to study the turbulence statistical characteristics and shock wave motion of STBLI, especially the low-frequency unsteadiness of STBLI. In general the study of wall-temperature effects on STBLI of compression corner is still not very sufficient.

In this research, we conduct numerical simulations of STBLI flows in a 24 deg compression corner with different wall temperatures, freestream Mach number 2.9, and Reynolds number 5881.4/mm. The ratios of wall temperature to recovery temperature $T_{w} / T_{r}$ are $0.6,1.14$, 1.4 , and 2.0, respectively. The flow parameters with $T_{w} / T_{r}=1.14$ are close to Bookey et al.'s experiment [14]. Based on the numerical data, the effect of wall temperature on the length of the separation bubble is analyzed and a semitheoretical formula is then given. The turbulent kinetic energy budgets are also analyzed in this paper. 


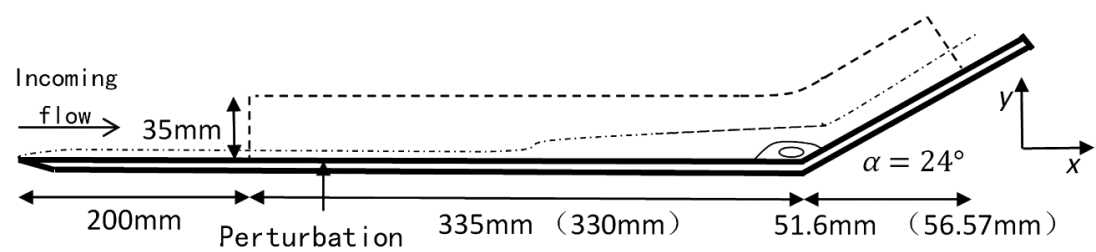

Fig. 1 Schematic diagram of numerical simulation setup.

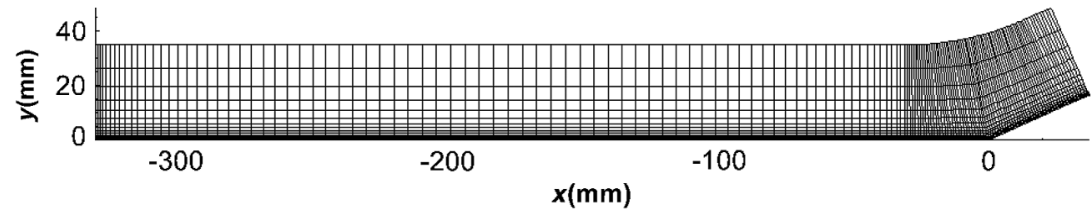

Fig. 2 Schematic diagram of computational meshes.

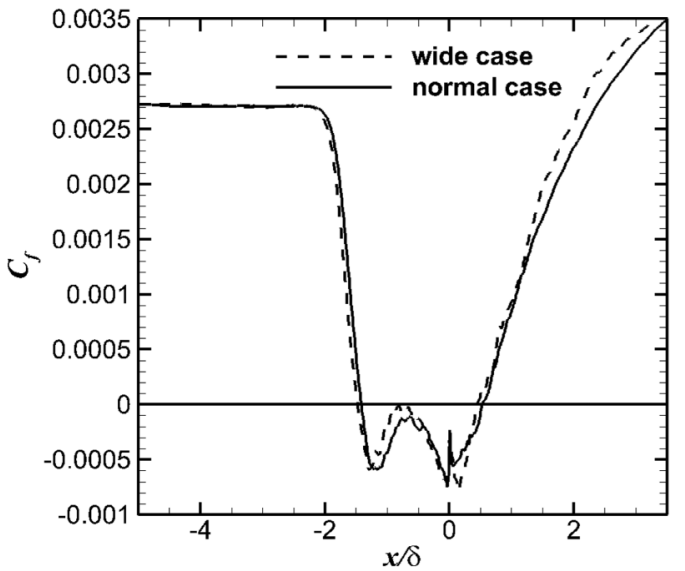

a) $T_{w} / T_{r}=0.6$

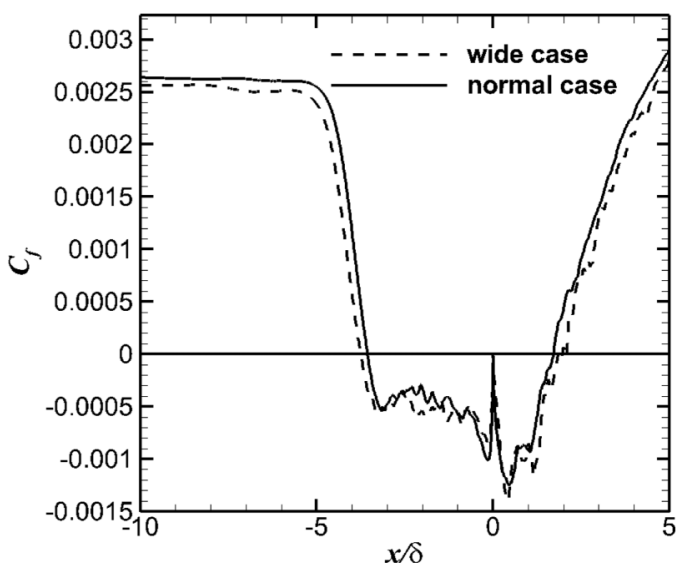

b) $T_{w} / T_{r}=2.0$

Fig. 3 Skin fraction coefficient of wide and normal cases.

\section{Simulation Setup}

\section{A. Numerical Simulation Setup and Computational Meshes}

The computing model used is supersonic flow over a $24 \mathrm{deg}$ corner, the same as Bookey et al.'s experimental model [14]. Figure 1 is the schematic diagram of the computing model, in which the corner is set at $x=0$. The dotted area in Fig. 1 is the computational domain. The inlet of the computational domain is located at the $200 \mathrm{~mm}$ downstream leading edge of the flat plate, and the inlet boundary condition is set by using the laminar profiles of flat-plate boundarylayer flow. Figure $\underline{2}$ shows the demonstration of mesh in the $x-y$ plane, in which the mesh is refined in the corner region to ensure the calculation accuracy. Table 1 shows the grid number and grid spacing in wall units for the four computing cases, in which $T_{w} / T_{r}$ represents the ratio of wall temperature to recovery temperature. Here, the recovery temperature is defined as $T_{r}=T_{\infty}\left[1+r(\gamma-1) M a^{2} / 2\right]$ and the temperature recovery coefficient is set as $r=0.89$.

For the first case, a cooling wall case with $T_{w} / T_{r}=0.6$, the computational mesh contains $3000 \times 200 \times 200$ points (streamwise $\times$ wall normal $\times$ spanwise), and the meshes for the other three cases contain $2160 \times 160 \times 140$. We use more grid points in the cooling wall case, because the actual Reynolds number (defined by the near-wall density and viscosity) for this case is much higher than that of the other three cases. The computational domain for all four cases is $L_{y}=$ $35 \mathrm{~mm}$ in the wall-normal direction and $0 \leq z \leq 14 \mathrm{~mm}$ in the

Table 1 Grid spacing in the wall unit

\begin{tabular}{lcccccc}
\hline \hline Case & Grid number & $\Delta x^{+}, x=-50 \mathrm{~mm}$ & $\Delta x^{+}, x \geqslant-25 \mathrm{~mm}$ & $\Delta y_{w}^{+}$ & $\Delta y_{\delta}^{+}$ & $\Delta z^{+}$ \\
\hline$T_{w} / T_{r}=0.6$ & $3000 \times 200 \times 200$ & 10.6 & 5.84 & 0.58 & 15.02 & 8.07 \\
$T_{w} / T_{r}=1.14$ & $2160 \times 160 \times 140$ & 6.20 & 4.26 & 0.49 & 10.17 & 4.86 \\
$T_{w} / T_{r}=1.4$ & $2160 \times 160 \times 140$ & 4.80 & 3.33 & 0.38 & 7.88 & 3.78 \\
$T_{w} / T_{r}=2.0$ & $2160 \times 160 \times 140$ & 3.12 & 2.07 & 0.24 & 5.19 & 2.44 \\
\hline \hline
\end{tabular}

Table 2 Flow parameters

\begin{tabular}{lcccccccc}
\hline \hline Case & $M_{\infty}$ & $R e_{\infty} / \mathrm{mm}$ & $T_{\infty}, \mathrm{K}$ & $T_{w}, \mathrm{~K}$ & $\delta, \mathrm{mm}$ & $\delta^{*}, \mathrm{~mm}$ & $\theta, \mathrm{mm}$ & $\Delta t$ \\
\hline$T_{w} / T_{r}=0.6$ & 2.9 & 5581.4 & 108.1 & 162.2 & 6.43 & 1.7 & 0.5 & 0.005 \\
$T_{w} / T_{r}=1.14$ & 2.9 & 5581.4 & 108.1 & 307.0 & 6.75 & 2.15 & 0.425 & 0.008 \\
$T_{w} / T_{r}=1.4$ & 2.9 & 5581.4 & 108.1 & 378.4 & 6.8 & 2.33 & 0.41 & 0.008 \\
$T_{w} / T_{r}=2.0$ & 2.9 & 5581.4 & 108.1 & 540.5 & 6.95 & 2.69 & 0.38 & 0.01 \\
Bookey's date [14] & 2.9 & 5581.4 & 108.1 & 307.0 & 6.7 & 2.36 & 0.43 & - \\
\hline \hline
\end{tabular}




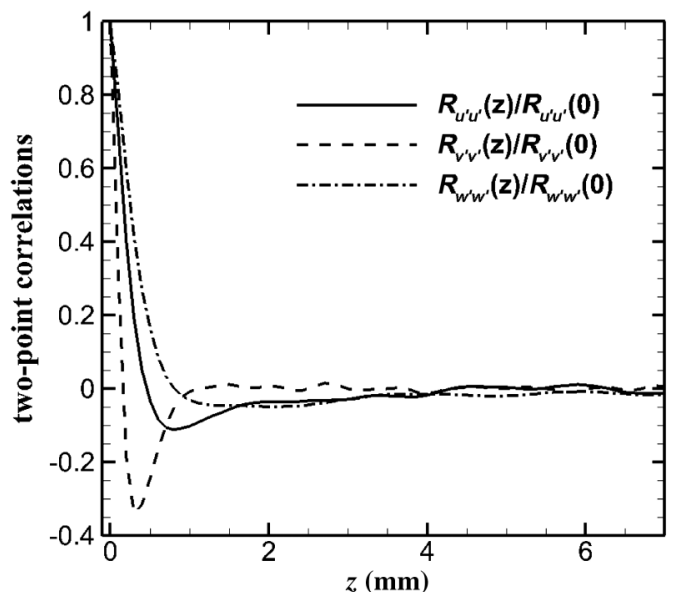

a) $y^{+}=10$

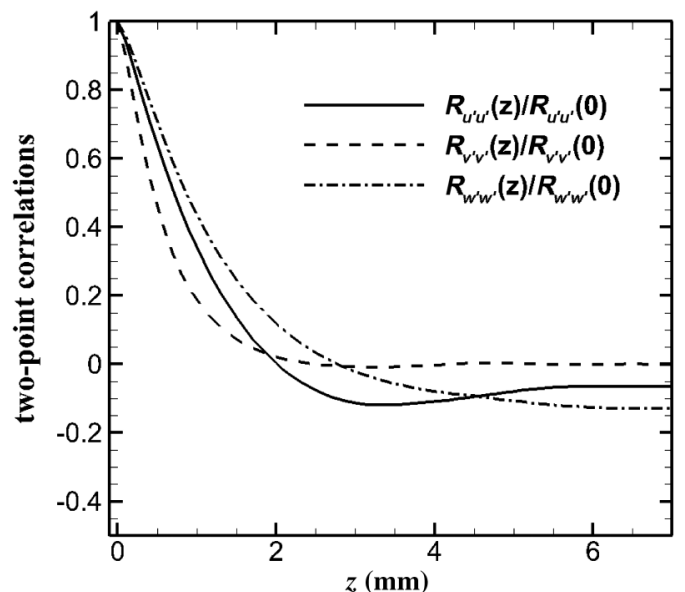

b) $y^{+}=198$

Fig. 4 Two-point correlations of velocity in flat-plate region (at $x=-30 \mathrm{~mm}$ ).

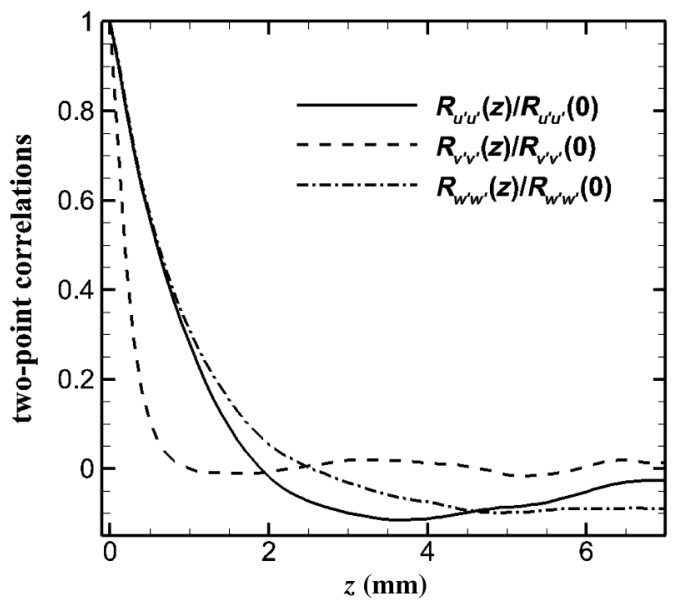

a) $y^{+}=10$

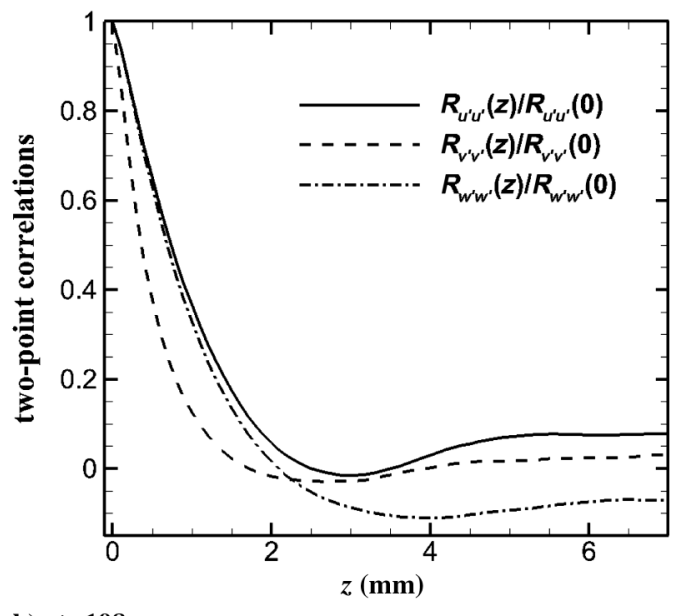

b) $y^{+}=198$

Fig. 5 Two-point correlations of velocity in separation region (at $x=-8 \mathrm{~mm}$ ).

spanwise direction, and the streamwise domain is $-330 \leq x \leq$ $56.57 \mathrm{~mm}$ for the first case and $-335 \leq x \leq 51.6 \mathrm{~mm}$ for the other three cases. The grid spacing in the flat-plate region, as well as that in the corner region, is also shown in Table 1 , in which all grid spaces are normalized by the wall unit at $x=-50 \mathrm{~mm}$ (flat-plate region). The wall-normal grid space of the first grid above the wall is denoted by

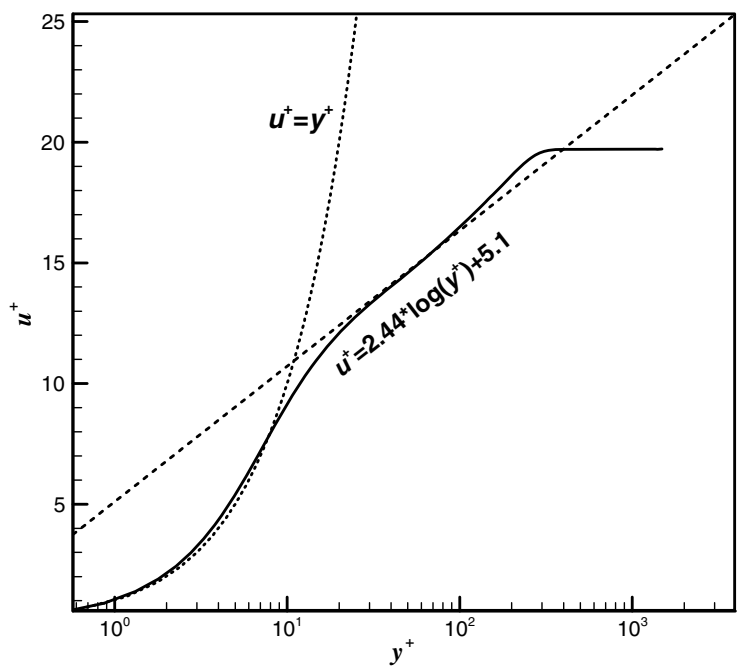

Fig. 6 Plot of mean van Driest velocity in flat-plate region of case $T_{w} / T_{r}=1.14$.
$\Delta y_{w}^{+}$, and the wall-normal grid space at the edge of boundary layer $(y=\delta)$ is denoted by $\Delta y_{\delta}^{+}$. In the corner region, the streamwise grid spacing is much smaller than that in the upstream flat-plate region to resolve the small scales of STBLI and separation flows. This table shows that the grid resolution in the flat-plate region is fine enough for the simulation of nonseparated flat-plate boundary layer [15-17].

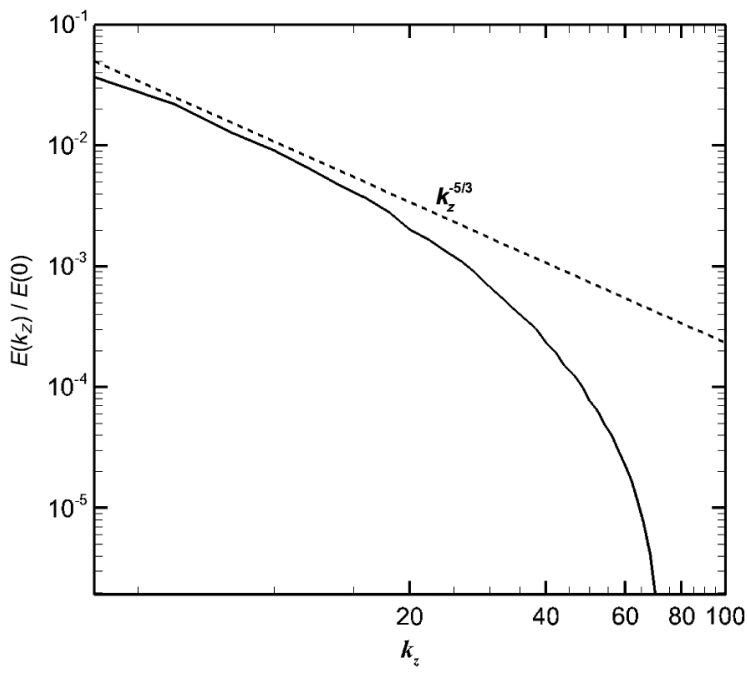

Fig. 7 Spanwise turbulent kinetic energy spectrum $\left(y^{+}=232\right)$. 


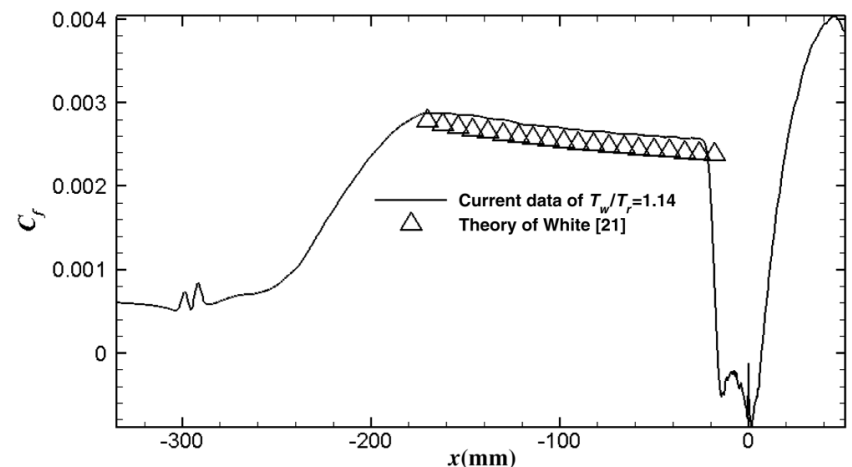

Fig. 8 Distribution of skin friction coefficient and its comparison with theoretical value.

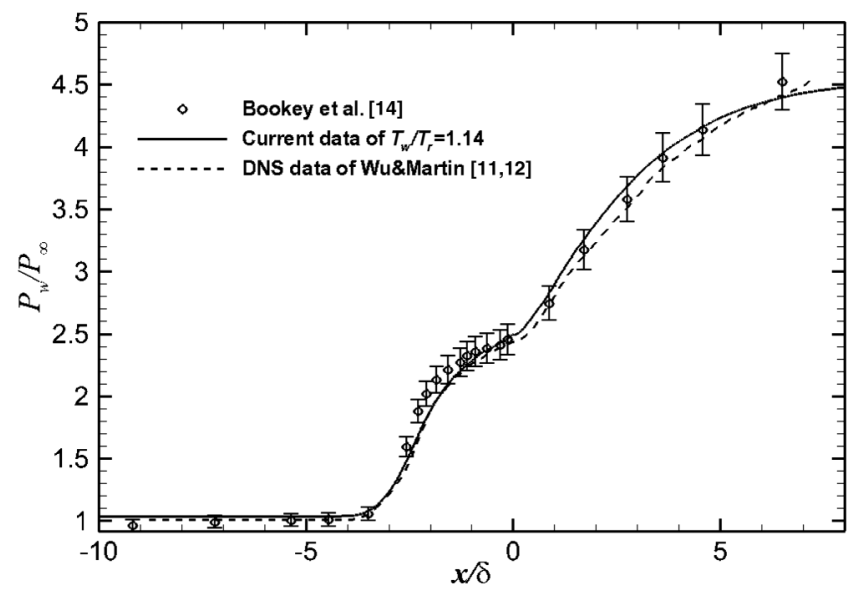

Fig. 9 Distribution of mean wall pressure for case $T_{w} / T_{r}=1.14$.

To trigger the transition, we impose the blow-and-suction perturbation $[13,17,18]$ on the wall in $-305 \leq x \leq-285 \mathrm{~mm}$. The amplitude of the perturbation is set as $A=0.1$ to trigger the bypass-type transition, and the perturbation contains multifrequency waves with five frequencies $\left(f_{0}, 2 f_{0}, 3 f_{0}, 4 f_{0}\right.$, and $\left.5 f_{0}\right)$, in which the nondimensional fundamental frequency is $f_{0}=0.1$ (approximately $96 \mathrm{kHz}$ ).

\section{B. Numerical Methods and Simulation Parameters}

The flow parameters are listed in Table 2. The freestream Mach number is 2.9, freestream Reynolds number per unit millimeter is 5581.4 , and freestream temperature is $108.1 \mathrm{~K}$, which are the same parameters as those used in Bookey et al.'s experiment [14]. The wall temperatures for the four computational cases are 162.2, $\overline{307}, 374$, and $540.5 \mathrm{~K}$, respectively. The computational parameters in the case $T_{w} / T_{r}=1.14$ are identical to Bookey et al.'s experiment parameters, and this case is also used as a test case to validate the computation. The perfect gas assumption is taken in computing, and Sutherland's law is used to compute the viscosity. The boundary-layer thickness $\delta$, boundary-layer displacement thickness $\delta^{*}$, and boundary-layer momentum thickness $\theta$ are also given in Table 2 . The three boundary-layer thicknesses of the current results are close to those of Bookey et al.'s experiment.

Navier-Stokes equations in the curvilinear coordinates are solved numerically without any turbulent model being used. The numerical dissipation plays the role of a subgrid-scale model. Therefore, this simulation can be referred to as an implicit large-eddy simulation or as an effective direct numerical simulation.

A high-resolution finite difference computational fluid dynamics code OpenCFD-SC [13] developed by our research group is used for the simulation, and the code is programmed by using MPI-Fortran 90. Steger-Warming splitting is used for the inviscous terms and then solved by using the fourth-order bandwidth-optimized weighted essentially non-oscillatory scheme (WENO-SYMBO) of $\mathrm{Wu}$ and Martín [11] and Martín et al. [19]. Viscous terms are discretized by using the eighth-order central scheme. The third-order TVD-type Runge-Kutta method [20] is used for time advance, and the CourantFriedrichs-Lewy number is between 0.1 and 0.2 for each case. Total time step is $233,000-365,000$ for the four cases. It takes about 10,00014,000 iterations to get statistically steady. Then, we record the flowfield for every 100 iterations and use it for statistics.

The code is parallel run on $240 \mathrm{CPU}$ cores of the TIANHE- 1 supercomputer in the National Supercomputer Center in Tianjin. The average wall time per one time step is about 2.05-2.2 s for the hot-wall cases $\left(T_{w} / T_{r}=1.14,1.4,2.0\right)$ and it is about $5.5 \mathrm{~s}$ for the cold-wall case ( $T_{w} / T_{r}=0.6$ ), because the grid number is much larger for this case.

\section{Numerical Results}

\section{A. Numerical Validation}

To validate that the computational domain in the spanwise direction for our numerical cases is wide enough, we double the

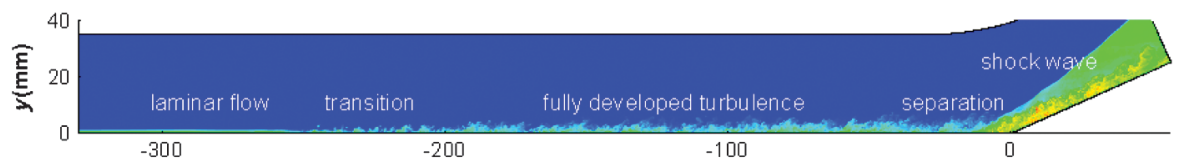

a) $T_{w} / T_{r}=0.6$

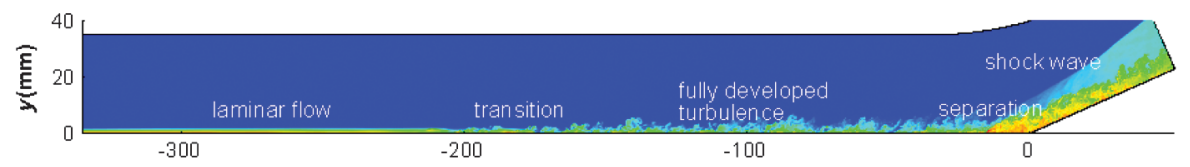

b) $T_{w} / T_{r}=1.14$

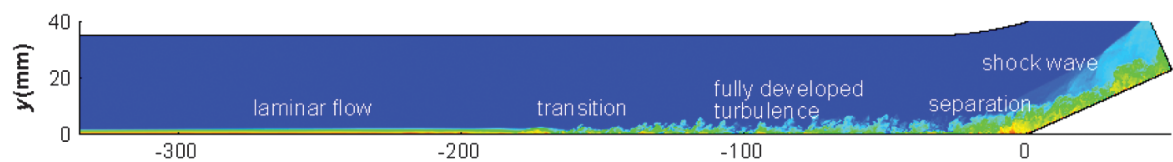

c) $T_{w} / T_{r}=1.4$

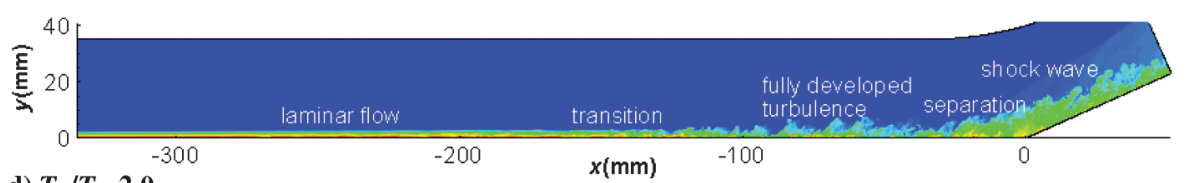

d) $T_{w} / T_{r}=2.0$

Fig. 10 Distribution of instantaneous temperature at midspan. 
computation domain in the spanwise direction of two cases $T_{w} / T_{r}=$ 0.6 and $T_{w} / T_{r}=2.0$ (the coldest case and the hottest one), and the grid width in the spanwise direction is kept the same. Figure 3 shows the skin friction coefficient $C_{f}$ of the normal case (14 mm spanwise domain) and the wide case (28 $\mathrm{mm}$ spanwise domain) for both $T_{w} / T_{r}=0.6$ and $T_{w} / T_{r}=2.0$. This figure shows that the difference between the normal and the wide cases is not obvious. The difference of separation bubble size is approximately $1.5 \%$ for the low-wall-temperature case and approximately 5\% for the high-walltemperature case. This shows that the spanwise computation domain is wide enough. Comparing Fig. $3 \mathrm{~b}$ with Fig. $3 \mathrm{a}$ shows that the highwall-temperature case is more sensitive to the spanwise domain size, the reason being that both the separation bubble size and the boundarylayer thickness are larger for the high-wall-temperature case, and so it is more sensitive to the spanwise domain size.

To further test the spanwise domain, we computed the two-point correlations for the case $T_{w} / T_{r}=1.14$ with spanwise domain $L z=14 \mathrm{~mm}$ (normal case). Figures $\underline{4}$ and $\underline{5}$ show the two-point correlations of $u, v$, and $w$ in the spanwise direction of the flat-plate region and the separation region, respectively. Two different normal locations of $y^{+}=10$ and $y^{+}=198(y \approx 0.6 \delta)$ are chosen. For all cases, a similar trend is observed, and the value of correlation keeps small values (between -0.1 and 0.1 ) at the region $z>3 \mathrm{~mm}$, which is small enough to be statistically independent. Figures $\underline{3}-\underline{5}$ show that the spanwise computational domain is wide enough.

Figure $\underline{6}$ plots the van Driest transformed velocity in the flat-plate region of case $T_{w} / T_{r}=1.14$. The velocity profile agrees well with the log law $u^{+}=2.44 \times \log \left(y^{+}\right)+5.1$ in the logarithmic region, which has the same constant $(C=5.1)$ as that used by Wu and Martín [11,12].

Figure 7 shows the one-dimensional spanwise turbulent kinetic energy spectrum [17] at the flat-plate region and $y^{+}=232(y \approx 0.7 \delta)$ of case $T_{w} / T_{r}=1.14$. The figure shows that all spectra exhibit a dropoff at about four decades and have a $-5 / 3$ slope, which indicates that it is the inertial subrange here and the turbulent boundary layer is in equilibrium. Because the Reynolds number is not very high, the $-5 / 3$ region is not long.

Figure 8 shows the distribution of skin friction coefficient $C_{f}$ for the case $T_{w} / \bar{T}_{r}=1.14$. This figure shows that $C_{f}$ has a drastic increase near the region $x=-200 \mathrm{~mm}$, which denotes the occurrence of the transition. $C_{f}$ gets its peak value at $x=-250 \mathrm{~mm}$ and decreases smoothly, indicating that the turbulence becomes fully developed. $C_{f}$ goes down rapidly downstream $x=-30 \mathrm{~mm}$ and then shows a negative value, indicating that the separation occurs. Downstream of the corner at $x=0 \mathrm{~mm}, C_{f}$ goes up rapidly and shows positive value again, indicating the reattachment of the flow. The triangles in this figure denote the theoretical value of $C_{f}$ by the formulation in [17,21], which agrees well with the data.

Figure 9 shows the distribution of the mean wall pressure $\bar{p}_{w} / p_{\infty}$ as a function of $x / \delta$ for the case $T_{w} / T_{r}=1.14$. The dashed line shows the DNS data of Wu and Martín $[11,12]$. The symbols indicate Bookey et al.'s experimental results [14], and the error bar is set as $5 \%$. This figure shows that our numerical data agree with both experimental results and the DNS data of Wu and Martín [11,12], which validates the computation. The current simulation predicts slightly higher wall pressures downstream of the pressure plateau comparing with the $\mathrm{Wu}$ and Martín data. It may be caused by the different grid distribution and transition method. More validations can be find in previous work [13].

\section{B. Flow Visualization}

Figure 10 shows the two-dimensional contour of the instantaneous temperature at the midspan of four cases of different wall temperatures. Regions of laminar flow, transition, fully developed turbulence, flow separation, and shock wave and boundary-layer interactions are shown clearly in this figure. This figure shows clearly that the transition is postponed with the increase of the wall temperature, and the reason is that the actual Reynolds number (defined by the near-wall density and viscosity) decreases obviously with the increase of wall temperature.
Figure 11 is the locally enlarged plot of instantaneous temperature in the corner region with the contour line of $u=0$. Shock waves and the interaction of shock wave/turbulence boundary layer are shown clearly in the figure. Separation bubbles appear downstream of the shock in all cases, and temperature peaks in the separation bubble. Figures 11a-11d also show that the size of the separation bubble increases obviously with the increase of wall temperature. Contour lines of instantaneous streamwise velocity $u=0$ are plotted in Figs. $11 \mathrm{a}-11 \mathrm{~d}$, and these lines can be regarded as the edge of the instantaneous separation bubbles. These lines show that the separation bubble is not always observed as one large bubble, but rather can be a series of smaller bubbles.

Figure 12 shows the visualization of coherent structures by using the isosurface of the second invariant of velocity gradient tensor $Q$ colored by the local streamwise velocity. We chose a low-walltemperature case with $T_{w} / T_{r}=0.6$ and a hot-wall-temperature case

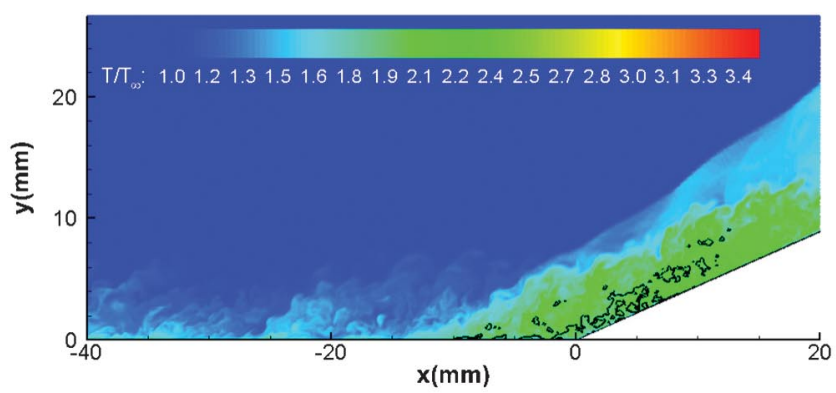

a) $T_{w} / T_{r}=0.6$

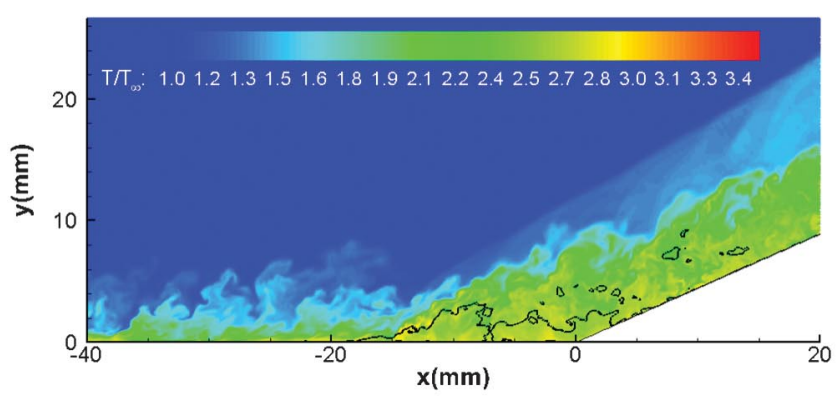

b) $T_{w} / T_{r}=1.14$

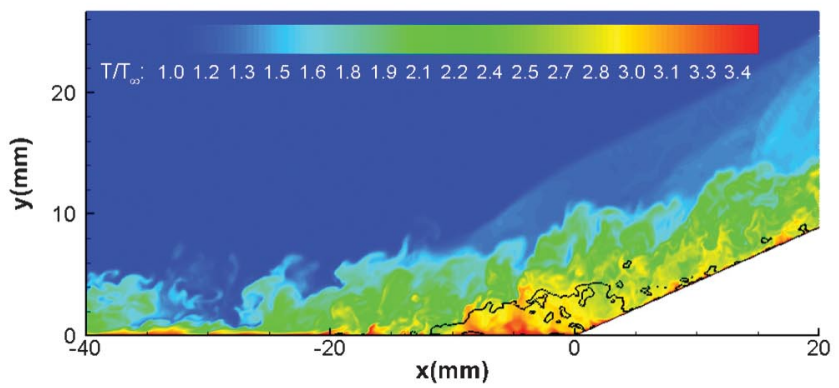

c) $T_{w} / T_{r}=1.4$

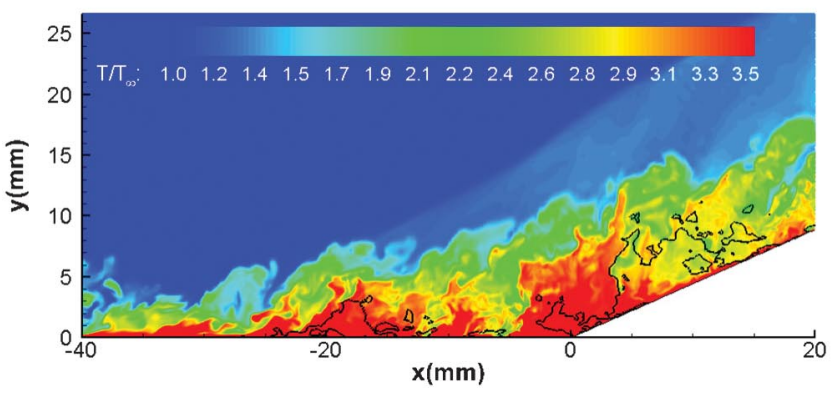

d) $T_{w} / T_{r}=2.0$

Fig. 11 Locally enlarged plot of instantaneous temperature with contour line of $u=0$. 


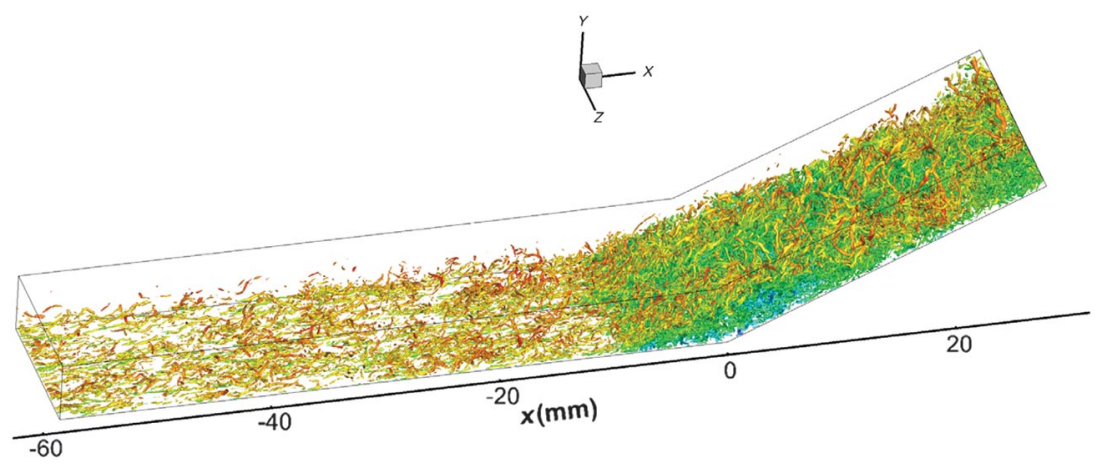

a) $T_{w} / T_{r}=0.6$

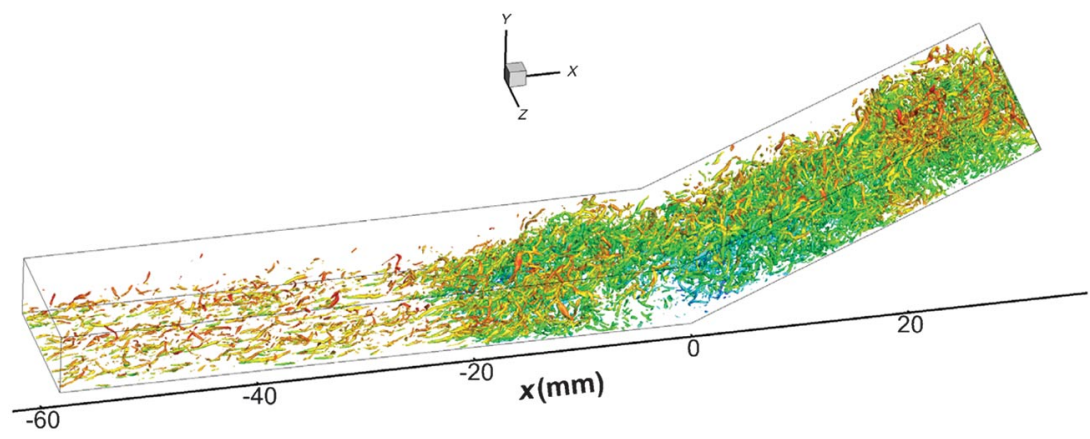

b) $T_{w} / T_{r}=1.4$

Fig. 12 Isosurface of second invariant of velocity gradient $Q$.

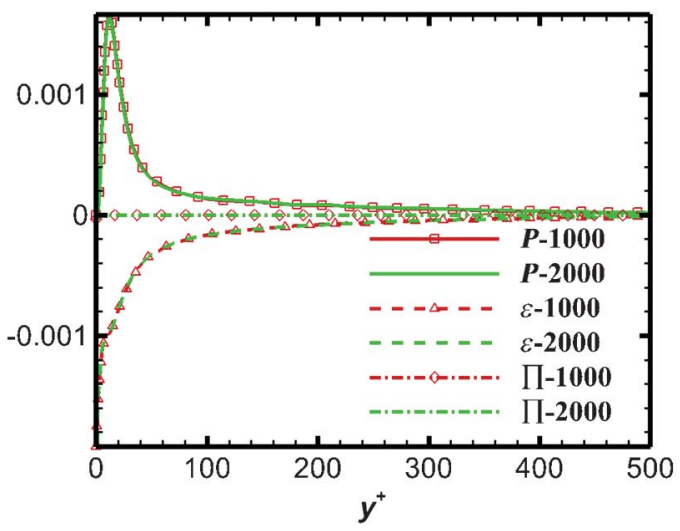

a) Before shock wave

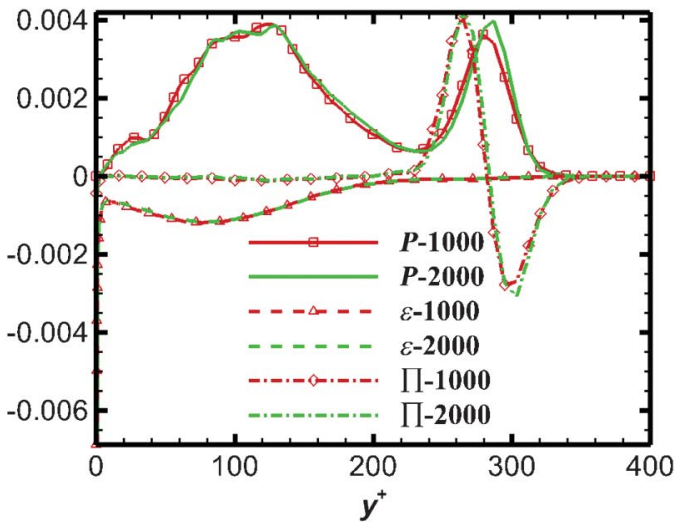

b) In separation region

Fig. 13 Time convergence study for $T_{w} / T_{r}=0.6$ case: 1000 vs 2000 samples.

with $T_{w} / T_{r}=1.4$ in this figure. The figure shows the cluster of coherent structures in the upstream turbulence boundary layer, and there are more clusters of coherent structures in the low-walltemperature case than in hot-wall-temperature one. The coherent structures are more chaotic downstream of the shock than those upstream of the shock. The results in Fig. 12 are consistent with the results by Duan et al. [22].

\section{Table 3 Expression of terms in TKE budget equation}

\begin{tabular}{lc}
\hline \hline Expression & Meanings \\
\hline$K=1 / \overline{\rho u_{i}^{\prime \prime} u_{i}^{\prime \prime}} / \bar{\rho}$ & TEK \\
$C=\partial\left(\bar{\rho} \tilde{u_{j}}\right) / \partial x_{j}$ & Convection \\
$P=-\overline{\rho u_{i}^{\prime \prime} u_{j}^{\prime \prime}} \partial \tilde{u}_{j} / \partial x_{j}$ & Production \\
$\varepsilon=\overline{\sigma_{i j}^{\prime} \partial u_{i}^{\prime \prime} / \partial x_{j}}$ & Dissipation \\
$T=-\partial\left(1 / 2 \overline{\rho u_{i}^{\prime \prime} u_{i}^{\prime \prime} u_{j}^{\prime \prime}}+\overline{p^{\prime} u_{j}^{\prime \prime}}\right) / \partial x_{j}$ & Turbulent transport \\
$D=\overline{\partial u_{i}^{\prime \prime} \sigma_{i j}^{\prime}} / \partial x_{i}$ & Viscous diffusion \\
$\Pi=\overline{p^{\prime} \partial u_{i}^{\prime \prime} / \partial x_{i}}$ & Pressure dilation \\
$M=\bar{u}_{i}^{\prime \prime}\left(\partial \bar{\sigma}_{i j} / \partial x_{i}-\partial \bar{p} / \partial x_{j}\right)$ & Due to density fluctuation \\
$\varepsilon_{d}=-\overline{\mu \partial u_{i}^{\prime \prime} / \partial x_{i} \partial u_{i}^{\prime \prime} / \partial x_{i}}$ & Dilation dissipation \\
\hline \hline
\end{tabular}

\section{Turbulent Kinetic Energy Budget}

Turbulent kinetic energy (TKE) is given by

$$
\tilde{k}=\frac{1}{2} \frac{\overline{\rho u_{i}^{\prime \prime} u_{i}^{\prime \prime}}}{\bar{\rho}}
$$

and its budget equation for compressible turbulence is

$$
\frac{\partial(\bar{\rho} k)}{\partial t}=-C+P+T+\Pi+D+M-\varepsilon
$$

The expression of each term is shown in Table $\underline{3}$. In this research, we pay attention to the production term $P$, dissipation term $\varepsilon$, pressuredilation term $\Pi$, and the dilation dissipation term $\varepsilon_{d}$. To test the statistical convergence for each term, we compared the statistics of 1000 samples and that of 2000 samples. Figure 13 shows the production term $P$, dissipation term $\varepsilon$, and pressure-dilation term $\Pi$ for the case $T_{w} / T_{r}=0.6$, in which Figs. $13 \mathrm{a}$ and $13 \mathrm{~b}$ plot those terms in the flat-plate and separation regions. In both of these regions, the 
curves with 1000 samples agree very well with the curves with 2000 samples, and thus we use 1000 samples for the following statistics.

Figure 14 shows the one-dimensional profiles of the production term $P$, dissipation term $\varepsilon$, pressure-dilation term $\Pi$, and the dilation dissipation term $\varepsilon_{d}$ of the four wall-temperature cases in the flat-plate region upstream of the shock. As shown in this figure, in the upstream region of the shock (flat-plate region), the turbulent production term reaches its peak in the buffer layer (the layer between the viscous sublayer and the logarithmic-law layer) and the dissipation term reaches its peak in the near-wall region. This figure also shows that both the pressure-dilation term and the dilation dissipation term are very small and ignorable for all four cases.

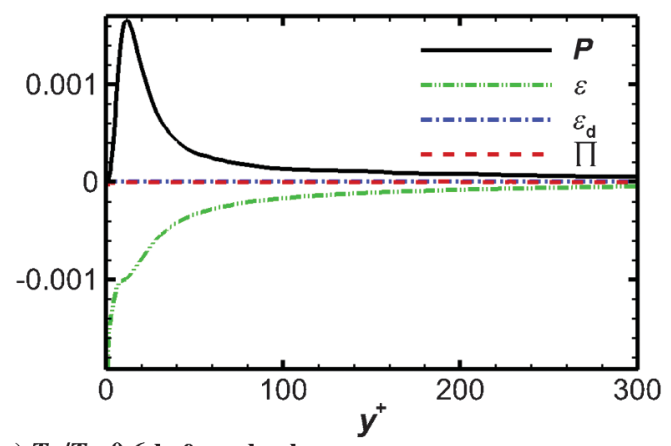

a) $T_{w} / T_{r}=0.6$ before shock wave

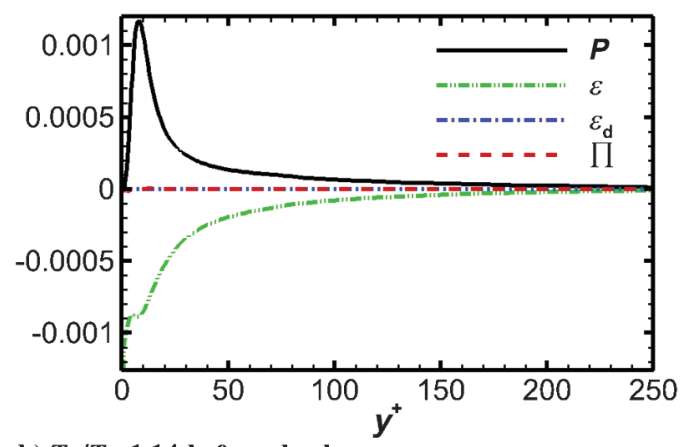

b) $T_{w} / T_{r}=1.14$ before shock wave

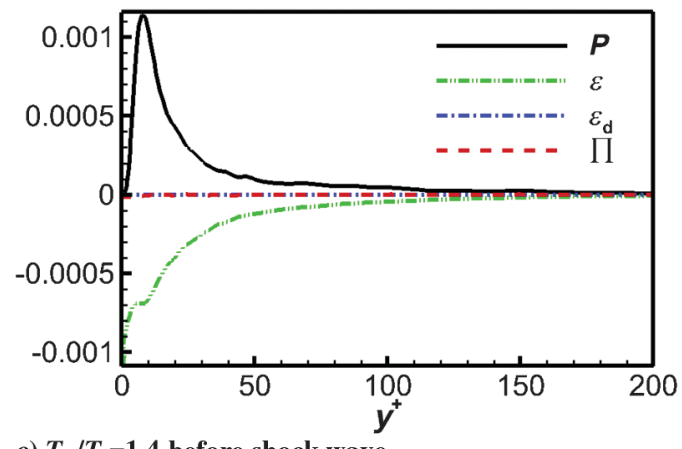

c) $T_{w} / T_{r}=1.4$ before shock wave

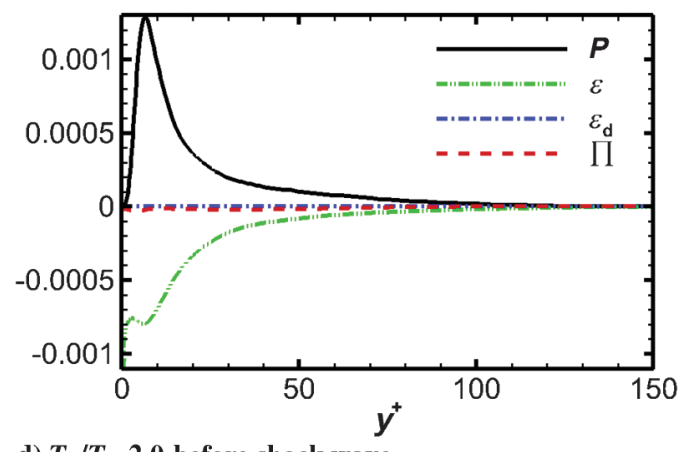

d) $T_{w} / T_{r}=2.0$ before shock wave

Fig. 14 Plots of four terms in TKE equation, in flat-plate region.
Figure 15 shows the profiles of $P, \varepsilon, \Pi$, and $\varepsilon_{d}$ in the separation bubble of the corner region. Different from those in the flat-plate region, the turbulent production terms show two peaks for all four cases: One peak is located in the boundary layer and the other is near the shock wave. This figure also shows that the high $P$ range is much larger than that in the flat-plate region, which means that total turbulent energy production is much higher in the separation bubble than that in the upstream flat-plate region. This figure also shows that the high $\varepsilon$ range is also much larger than that in the upstream flat-plate

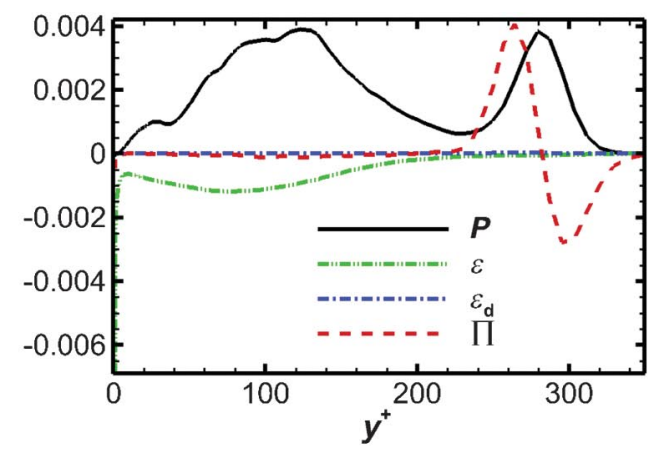

a) $T_{w} / T_{r}=0.6$ in separation region

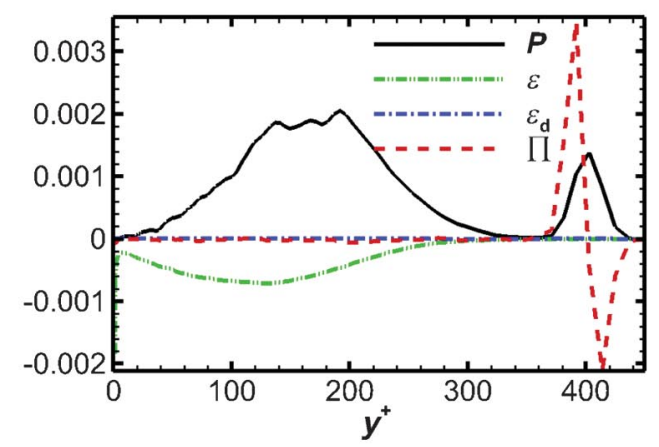

b) $T_{w} / T_{r}=1.14$ in separation region

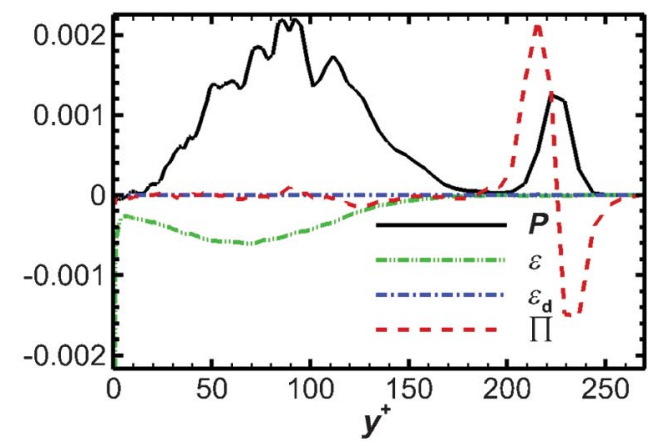

c) $T_{w} / T_{r}=1.4$ in separation region

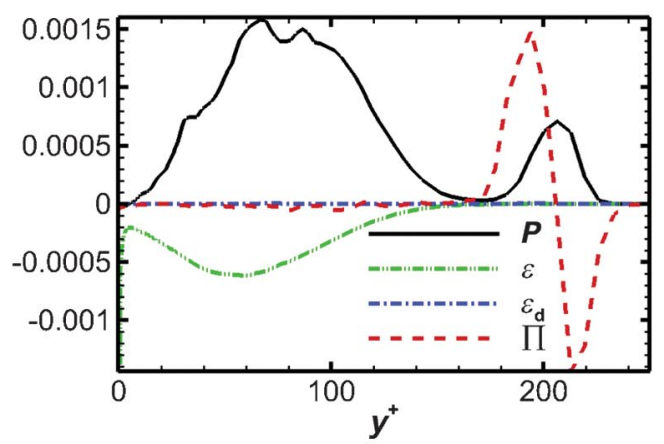

d) $T_{w} / T_{r}=2.0$ in separation region

Fig. 15 Plots of four terms in TKE equation, in separation region. 


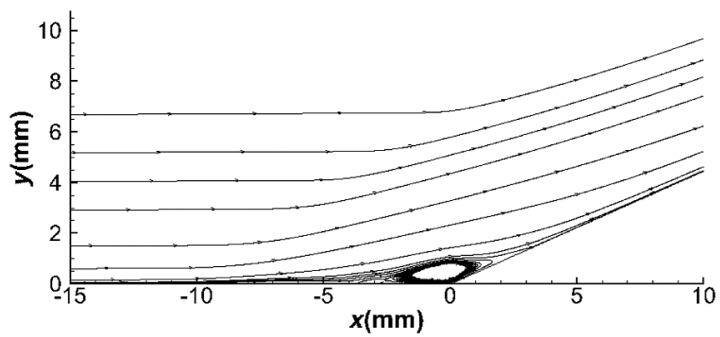

a) $T_{w} / T_{r}=0.6$

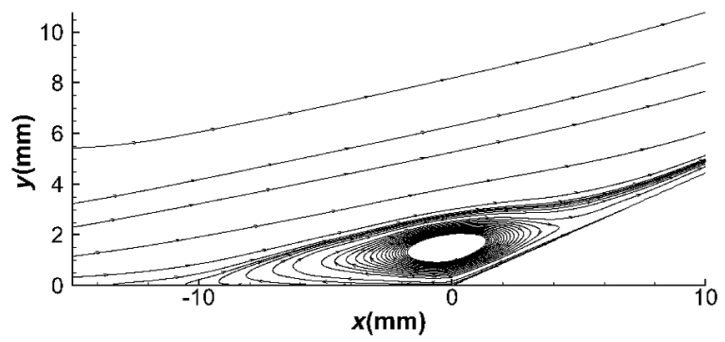

c) $T_{w} / T_{r}=1.4$

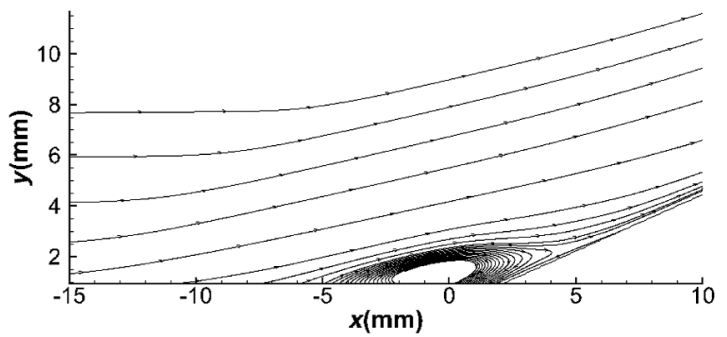

b) $T_{w} / T_{r}=1.14$

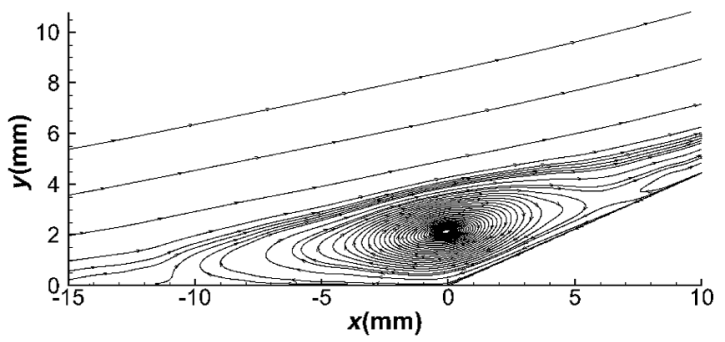

d) $T_{w} / T_{r}=2$

Fig. 16 Streamlines of mean flowfield in corner region.

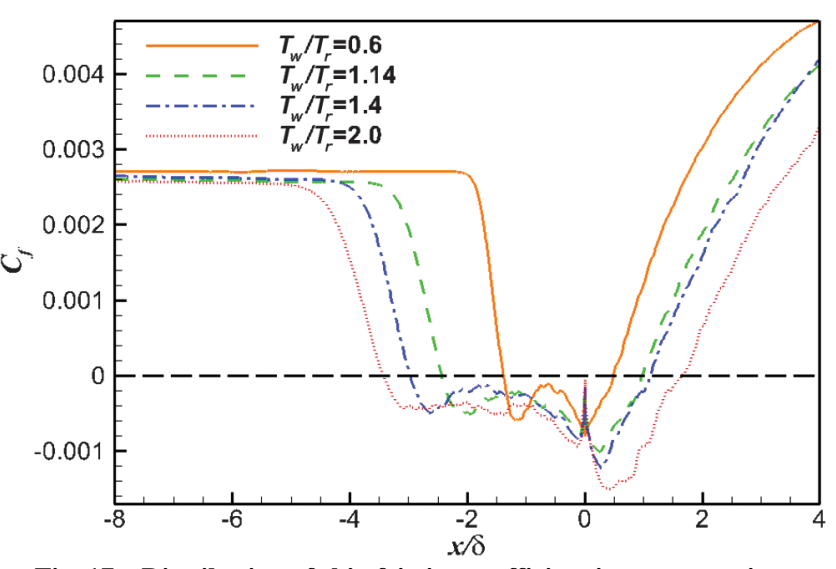

Fig. 17 Distribution of skin friction coefficient in corner region.

region in the separation bubble, which means that turbulence is very active in the separation bubble.

The pressure-dilatation term $\Pi$ and the dilation dissipation term $\varepsilon_{d}$ denote the intrinsic compressibility effect [23], and these two terms are also shown in Figs. 14 and 15 . These figures show that $\varepsilon_{d}$ keeps an ignorable value in all regions for all four cases. The pressure-dilation term $\Pi$ is also very small in all regions, except near the shock wave. This indicates that the effect of the intrinsic compressibility effect is ignorable in the region where it does not contain the main shock. Therefore, the authors conjecture that compressibility is not strongly important for these scenarios.

\section{Effect of Wall Temperature on Size of Separation Bubble}

\section{A. Size of Separation Bubble}

Figure 16 shows the streamline of the mean flow of the four cases. The mean flow is computed by using both spanwise and time

Table 4 Separation point, reattached point, and size of separation bubble

\begin{tabular}{lccc}
\hline \hline Case & $x_{s} / \delta$ & $x_{r} / \delta$ & $L / \delta$ \\
\hline$T_{w} / T_{r}=0.6$ & -1.38 & 0.49 & 1.87 \\
$T_{w} / T_{r}=1.14$ & -2.44 & 0.98 & 3.42 \\
$T_{w} / T_{r}=1.4$ & -2.79 & 1.12 & 4.09 \\
$T_{w} / T_{r}=2.0$ & -3.60 & 1.49 & 5.09 \\
\hline \hline
\end{tabular}

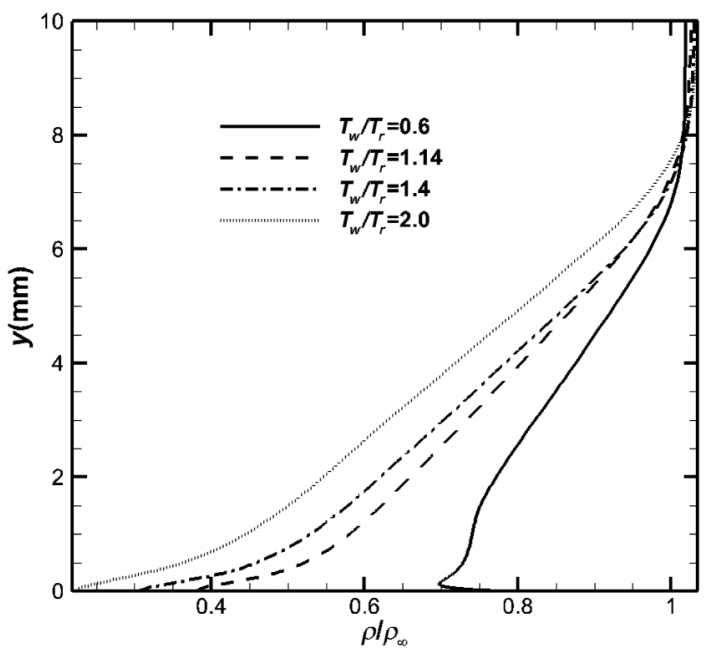

Fig. 18 Distribution of mean density in boundary layer.

averages. This figure clearly shows the separation bubble in the corner region and it also shows that the size of separation bubbles increase with the increase of wall temperature.

Figure 17 shows the distribution of the mean skin friction coefficient $C_{f}$ for the four wall-temperature cases in the corner region, where the horizontal coordinates are normalized by $\delta$. The size of separation bubble can be measured by the length of negative $C_{f}$ regions in this figure. The first zero point of $C_{f}$ is defined as the separation point and the second zero point is defined as the reattachment point. The scale of the separation bubble is defined as the length between separation point and reattachment point. The location of separation point $\left(x_{s} / \delta\right)$ and reattachment point $\left(x_{r} / \delta\right)$ and the size of separation bubble $(L / \delta)$ are shown in Table 4 . It shows that the length of separation bubble increases with the increase of wall temperature.

\section{B. Semitheoretical Formula for Effect of Wall Temperature on Length of Separation Bubbles}

As in the analysis in Sec. III, the intrinsic compressibility effect is not significant for all cases and it should not be the main reason of the increase of separation bubbles. The authors assume that wall temperature has an effect on the separation bubble size by changing the mean quantities of the flowfield, especially the mean density and mean viscosity. Figure 18 shows the distribution of mean density in the boundary layer of the four wall-temperature cases in the upstream 


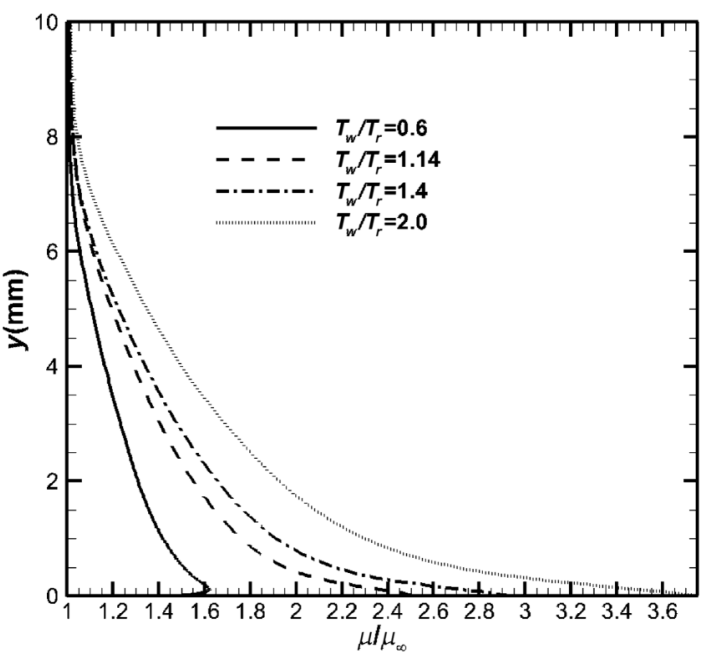

Fig. 19 Distribution of mean viscosity coefficient in boundary layer.

of the separation bubble, and this figure shows that near-wall density increases obviously with the decrease of wall temperature. Changes of density will affect the mass flow rate of the separation bubble and then affect the size of the separation bubble. Figure 19 shows the distribution of the mean viscosity coefficient in the boundary layer of the four cases at the same position as that in Fig. 18. Figure 19 shows that the mean viscosity coefficient increases obviously with the increase of wall temperature, especially in the near-wall region. Obviously, the viscosity coefficient is also a key effective factor on the size of separation bubbles.

Based on the analysis, the authors assume that wall temperature has an effect on size of the separation bubble by changing the mean density and mean viscosity in the near-wall region (i.e., it is a kind of Reynolds number effect). We define the actual Reynolds number by using mixed freestream and wall values:

$$
R e_{w}=\frac{\rho_{w} u_{\infty} L_{\infty}}{\mu_{w}}
$$

in which $u_{\infty}$ is the freestream velocity, $L_{\infty}$ is the reference length (e.g., $1 \mathrm{~mm}$ ), and $\rho_{w}$ and $\mu_{w}$ are the density and viscosity in the wall upstream the separation bubble, respectively. With fixed ramp angle and fixed freestream Mach number, the size of the separation bubble should be determined by $R e_{w}$.

According to Prandtl's boundary-layer theory, the pressure keeps approximately constant in the boundary layer. Considering as the state equation of perfect gas $p=\rho R T$, the wall density should be inversely proportional to the wall temperature:

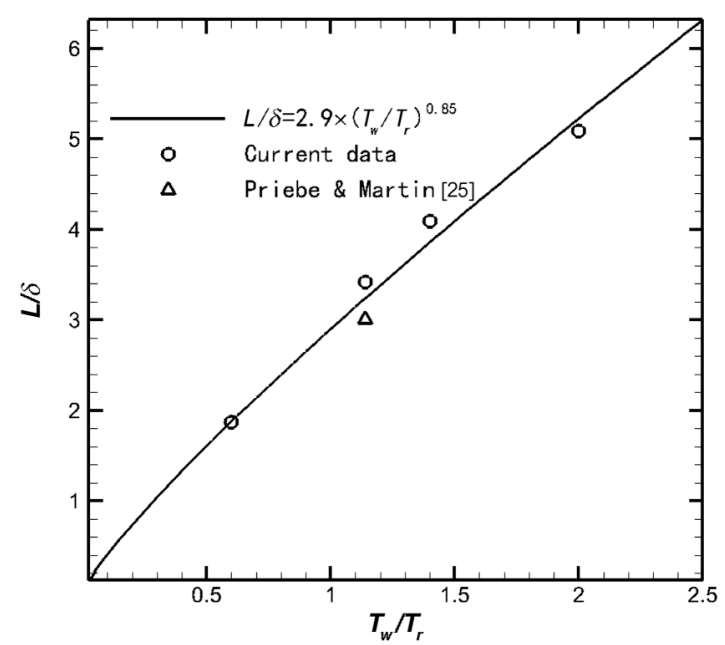

Fig. 20 Length of separation bubble as a function of wall temperature.

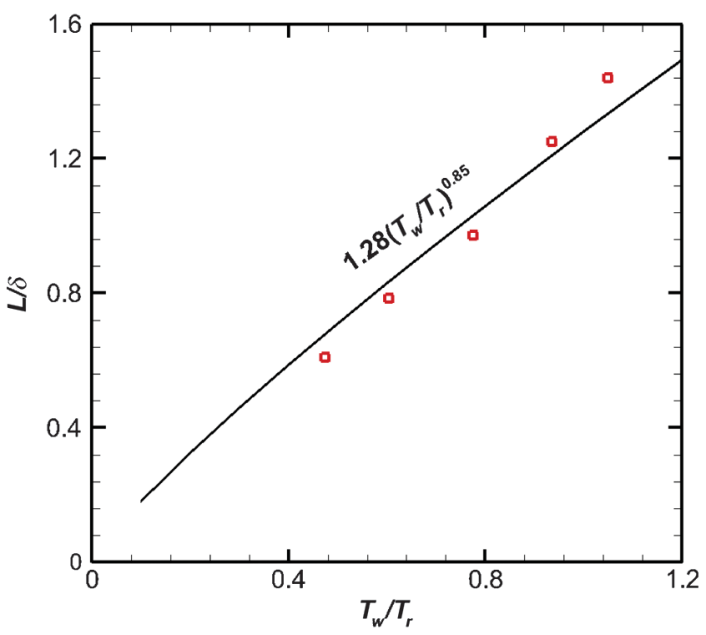

Fig. 21 Separation distance of Spaid and Frishett's experimental data [9].

$$
\rho_{w} \propto 1 / T_{w}
$$

The relationship between the air viscosity coefficient and temperature can be approximated as the following power law [24]:

$$
\mu \propto T^{0.7}
$$

Considering Eqs. (3-5), and that $u_{\infty}$ and $L_{\infty}$ are constant values here, we have

$$
R e_{w} \propto 1 / T_{w}^{17}
$$

We assume that, in the case of turbulent flow, the size of separation bubble varies with the upstream Reynolds number as the following rules:

$$
L / \delta \propto 1 / \sqrt{R e_{w}}
$$

Combining Eq. (6) with Eq. (ㄱ), we have

$$
L / \delta \propto \sqrt{T_{w}^{1.7}}=T_{w}^{0.85}
$$

Considering recovery temperature $T_{r}$ is a constant value here, we finally get the semitheoretical formula

$$
L / \delta \propto\left(T_{w} / T_{r}\right)^{0.85}
$$

That is, the separation bubble size is proportional to the 0.85 power of the wall temperature.

Figure 20 shows the length of the separation bubble as a function of wall temperature. The solid line denotes the semitheoretical formula (9), which is $L / \delta=2.9\left(T_{w} / T_{r}\right)^{0.85}$, and the circle symbols denote the current results. The delta symbols denote the result of Priebe and Martín [25] at the similar condition. This figure shows that our semitheoretical formula has a good predictive ability. This also validates that the assumption of Eq. (7) is correct.

Figure 21 shows the separation distance of Spaid and Frishett's experimental data [9], which is measured in a wind-tunnel experiment with a $20 \mathrm{deg}$ compression corner with Mach 2.9 freestream flow. The line in this figure denotes our semitheoretical formula $L / \delta=1.28\left(T_{w} / T_{r}\right)^{0.85}$ and it shows a good prediction. Because the corner angle ( $20 \mathrm{deg})$ is smaller than the preceding one ( $24 \mathrm{deg})$, the separation length is much smaller, and thus the coefficient 1.28 is also smaller than the preceding one (2.9).

\section{Conclusions}

Numerical simulation of STBLI flows in a 24 deg compression ramp is conducted to study the effect of wall temperature on the size 
of separation bubble. The freestream Mach number is 2.9 and the freestream Reynolds number is $5581.4 / \mathrm{mm}$. Four cases of different wall temperatures are simulated, and the ratios of wall temperature to recovery temperature $T_{w} / T_{r}$ are $0.6,1.14,1.4$, and 2.0 , respectively. The statistical results with $T_{w} / T_{r}=1.14$ are tested to validate the simulation, and the results show a good agreement with theoretical, experimental, and previous numerical simulating results. The effects of wall temperature on size of separation bubble are studied, and the turbulent kinetic energy budgets are also analyzed based on the simulation data. Conclusions are as follows:

1) Wall temperature has a direct effect on the size of separation bubble, and the size increases significantly with the increase of wall temperature.

2) Wall temperature appears to affect the size of separation bubble mainly by changing the near-wall density and viscosity.

3) Theoretical analysis leads to a semitheoretical formula to predict the size of separation bubble: $L / \delta \propto\left(T_{w} / T_{r}\right)^{0.85}$. The semitheoretical formula agrees well with the numerical data proposed in this paper.

4) Intrinsic compressibility effect is not significant in the cases studied here.

\section{Acknowledgments}

This work was supported by the National Science Foundation of China (91441103, 1372330, 11472279) and National key Research and Development program (2016YFA0401200). The authors thank the National Supercomputer Center in Tianjin, Supercomputing Center of Chinese Academy of Sciences, and Shandong Supercomputing Center for providing computer time. Thanks to Dexun Fu and Yanwen Ma, from the Institute of Mechanics, Chinese Academy of Sciences, for their suggestions. Thanks to the referees for their valuable suggestions and comments; it is also very helpful to our research.

\section{References}

[1] Dolling, D. S., "Fifty Years of Shock-Wave/Boundary-Layer Interaction Research: What Next?" AIAA Journal, Vol. 39, No. 8, 2001, pp. 1517 1531.

doi: $10.2514 / 2.1476$

[2] Clemens, N. T., and Narayanaswamy, V., "Low-Frequency Unsteadiness of Shock Wave/Turbulent Boundary Layer Interactions,", Annual Review of Fluid Mechanics, Vol. 46, No. 1, 2014, pp. 469-492.

[3] Gaitonde, D. V., "Progress in Shock Wave/Boundary Layer Interactions," Progress in Aerospace Sciences, Vol. 72, Jan. 2015, pp. 80-99. doi:10.1016/..paerosci.2014.09.002

[4] Larchevêque, L., Dupont, P., Martel, E. D., Garnier, E., and Debiève, J. F., Experimental and Numerical Study of Unsteadiness in Boundary Layer/Shock Wave Interaction/Turbulence and Interactions, SpringerVerlag, Berlin, 2010, pp. 263-269.

[5] Schülein, E., "Skin Friction and Heat Flux Measurements in Shock/ Boundary Layer Interaction Flows," AIAA Journal, Vol. 44, No. 8, 2006, pp. 1732-1741. doi: $10.2514 / 1.15110$

[6] Marini, M., "Analysis of Hypersonic Compression Ramp Laminar Flows Under Sharp Leading Edge Conditions," Aerospace Science and Technology, Vol. 5, No. 4, 2001, pp. 257-271. doi:10.1016/S1270-9638(01)01109-9

[7] John, B., and Kulkarni, V., "Numerical Assessment of Correlations for Shock Wave Boundary Layer Interaction," Computers and Fluids, Vol. 90, Feb. 2014, pp. 42-50. doi:10.1016/j.compfluid.2013.11.011

[8] Jaunet, V., Debiève, J. F., and Dupont, P., "Length Scales and Time Scales of a Heated Shock-Wave/Boundary-Layer Interaction," AIAA Journal, Vol. 52, No. 11, 2014, pp. 2524-2532. doi:10.2514/1.J052869
[9] Spaid, F. W., and Frishett, J. C., "Incipient Separation of a Supersonic, Turbulent Boundary Layer, Including Effects of Heat Transfer," AIAA Journal, Vol. 10, No. 7, 1972, pp. 915-922. doi: $10.2514 / 3.50245$

[10] Adams, N. A., "Direct Simulation of the Turbulent Boundary Layer Along a Compression Ramp at $M=3$ and $R e=1685$," Journal of Fluid Mechanics, Vol. 420, Oct. 2000, pp. 47-83. doi:10.1017/S0022112000001257

[11] Wu, M., and Martín, M. P., "Direct Numerical Simulation of Shockwave and Turbulent Boundary Layer Interaction Induced by a Compression Ramp," AIAA Journal, Vol. 45, No. 4, 2007, 879-889. doi:10.2514/1.27021

[12] Wu, M. W., and Martín, M. P., "Analysis of Shock Motion in Shockwave and Turbulent Boundary Layer Interaction Using Direct Numerical Simulation Data," Journal of Fluid Mechanics, Vol. 594, Jan. 2008, pp. 71-83. doi:10.1017/S0022112007009044

[13] Li, X. L., Fu, D. X., and Ma, Y. W., "Direct Numerical Simulation of Shock/Turbulent Boundary Layer Interaction in a Supersonic Compression Ramp," Science China Physics, Mechanics and Astronomy, Vol. 53, No. 9, 2010, pp. 1651-1658. doi:10.1007/s11433-010-4034-x

[14] Bookey, P. B., Wyckham, C., Smits, A., and Martin, M. P., "New Experimental Data of STBLI at DNS/LES Accessible Reynolds Numbers," AIAA Paper 2005-309, 2005.

[15] Liang, X., and Xinliang, L. I., "DNS of a Spatially Evolving Hypersonic Turbulent Boundary Layer at Mach 8," Science China Physics Mechanics and Astronomy, Vol. 56, No. 7, 2013, pp. 1408-1418. doi:10.1007/s11433-013-5102-9

[16] Georgiadis, N. J., Rizzetta, D. P., and Fureby, C., "Large-Eddy Simulation: Current Capabilities, Recommended Practices, and Future Research," AIAA Journal, Vol. 48, No. 8, 2010, pp. 1772-1784. doi:10.2514/1.J050232

[17] Pirozzoli, S., and Grasso, F., "Direct Numerical Simulation of Impinging Shock Wave/Turbulent Boundary Layer Interaction at $M=2.25$," Physics of Fluids, Vol. 18, No. 6, 2006, Paper 065113. doi:10.1063/1.2216989

[18] Rai, M. M., Gatski, T. B., and Erlebacher, G., "Direct Simulation of Spatially Evolving Compressible Turbulent Boundary Layers," AIAA Paper 1995-0583, 1995.

[19] Martín, M. P., Taylor, E. M., Wu, M., and Weirs, V. G., "A BandwidthOptimized WENO Scheme for the Effective Direct Numerical Simulation of Compressible Turbulence," Journal of Computational Physics, Vol. 220, No. 1, 2006, pp. 270-289. doi:10.1016/j.jcp.2006.05.009

[20] Jiang, G. S., and Shu, C. W., "Efficient Implementation of Weighted ENO Schemes," Journal of Computational Physics, Vol. 126, No. 1, 1996, pp. 202-228. doi:10.1006/jcph.1996.0130

[21] White, F. M., and Corfield, I., Viscous Fluid Flow, McGraw-Hill, New York, 2006, pp. 548-550.

[22] Duan, L., Beekman, I., and Martín, M. P., "Direct Numerical Simulation of Hypersonic Turbulent Boundary Layers. Part 3. Effect of Mach Number," Journal of Fluid Mechanics, Vol. 672, April 2011, pp. 245-267. doi:10.1017/S0022112010005902

[23] Morkovin, M. V., "Effects of Compressibility on Turbulent Flows," Mécanique de la Turbulence, edited by Favre, A., CNRS, Gordon and Breach, New York, 1962, pp. 367-380.

[24] Coleman, G. N., Kim, J., and Moser, R. D., "A Numerical Study of Turbulent Supersonic Isothermal-Wall Channel Flow," Journal of Fluid Mechanics, Vol. 305, Dec. 1995, pp. 159-183. doi:10.1017/S0022112095004587

[25] Priebe, S., and Martín, M. P., "Low-Frequency Unsteadiness in Shock Wave-Turbulent Boundary Layer Interaction," Journal of Fluid Mechanics, Vol. 699, May 2012, pp. 1-49.

doi: $10.1017 / \mathrm{jfm} .2011 .560$ 